\title{
Power Condition Maintenance Based on Internet of Things Technology
}

\author{
XU Shi-jun ${ }^{1}$ PEI Xu-liang ${ }^{2}$ LIU Jin-hui ${ }^{2}$ MAO Feng-chao ${ }^{2}$
}

\begin{abstract}
Objective: Compared with the general condition of power equipment, the advantages of state maintenance based on Internet of Things are obvious. Methods: First, the information collected by the Internet of Things is used to determine the maintenance plan, and then the application of the Internet of Things technology in the whole cycle management of the power equipment is introduced. Finally, the state maintenance and general maintenance based on the Internet of things are analyzed. Results: In addition to the maintenance of more efficient intelligent maintenance, information and data more secure and reliable, but also improve the efficiency of maintenance work, reducing the waste of human resources and so on. Conclusion: Because of the obvious advantages of power equipment condition maintenance based on Internet of Things technology, it has important practical significance and application value to carry on the thorough research.
\end{abstract}

Key words: Internet of things technology; power equipment; state maintenance

\section{Introduction}

Power equipment state maintenance work can accurately grasp the equipment working conditions, life, etc., and timely detection of equipment failure or abnormal problems, to prevent power system power and other issues. Based on the state of the Internet technology in addition to the maintenance of more efficient maintenance of intelligent, more secure and reliable information, but also improve

1 China National Offshore Oil Corporation Qinhuangdao 32-6 operating company Tianjin 300452 the maintenance efficiency and reduce the waste of human resources. In this paper, the information collected by the Internet of Things is used to determine the maintenance plan, and then the application of the Internet of Things technology in the whole cycle management of the power equipment is introduced. Finally, the state maintenance and general maintenance based on the Internet of Things technology are analyzed and compared.

1. Determine the maintenance plan by combining the information collected by the Internet of Things

1.1 Information analysis and maintenance program development

The so-called Internet of Things technology, mainly for the object is the information collection, information analysis and maintenance program development, should start from the overall point of view, and the basic work is directly related. As a result of the management objectives of the different, but also build a corresponding management system, in the market, technical research at the same time, to determine the state maintenance of multi-strategy management system. And set up expert service function, the reason is to ensure the safety level in the standard range, improve the power equipment maintenance program.

\subsection{Information processing analysis}

For information collected through the Internet of Things technology, to deal with the timely analysis, the specific process is:

$\square$ The cause of the failure study;

Real-time focus on the detection of key information collection;

Collection, analysis and storage of test data;

Setting and adjustment of fault parameter value; 


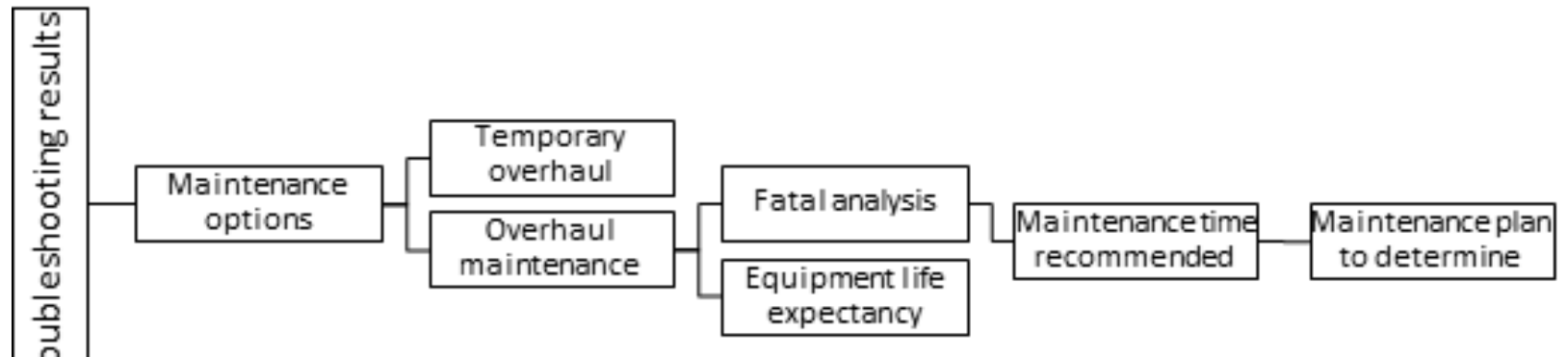

Figure 1 State maintenance program to simplify the work diagram

According to the fault classification standard, clear the fault category.

1.3 According to the analysis results to determine the maintenance program

On the Internet of things data analysis results, the specific follow-up is:

Warning information processing. Combine the warning information in time and effectively deal with the relevant analysis results. According to the previous case and the working condition of the electric power equipment, the overall judgment of the actual situation of the electric power equipment, clear the objective accuracy of the warning message, and then clear the power equipment is a normal working state or fault state.

Accurate and objective information to judge. Effectively use the main station monitoring role, its monitoring equipment to send work instructions, and then according to the information being monitored by the information to determine the accuracy of the warning message objectivity.

Fault judgment expert service, that is, combined with the main equipment of the power system warning information and auxiliary equipment warning information, analysis and judgment. And the results of the analysis need to verify the staff, according to the maintenance staff of the work experience, decide whether

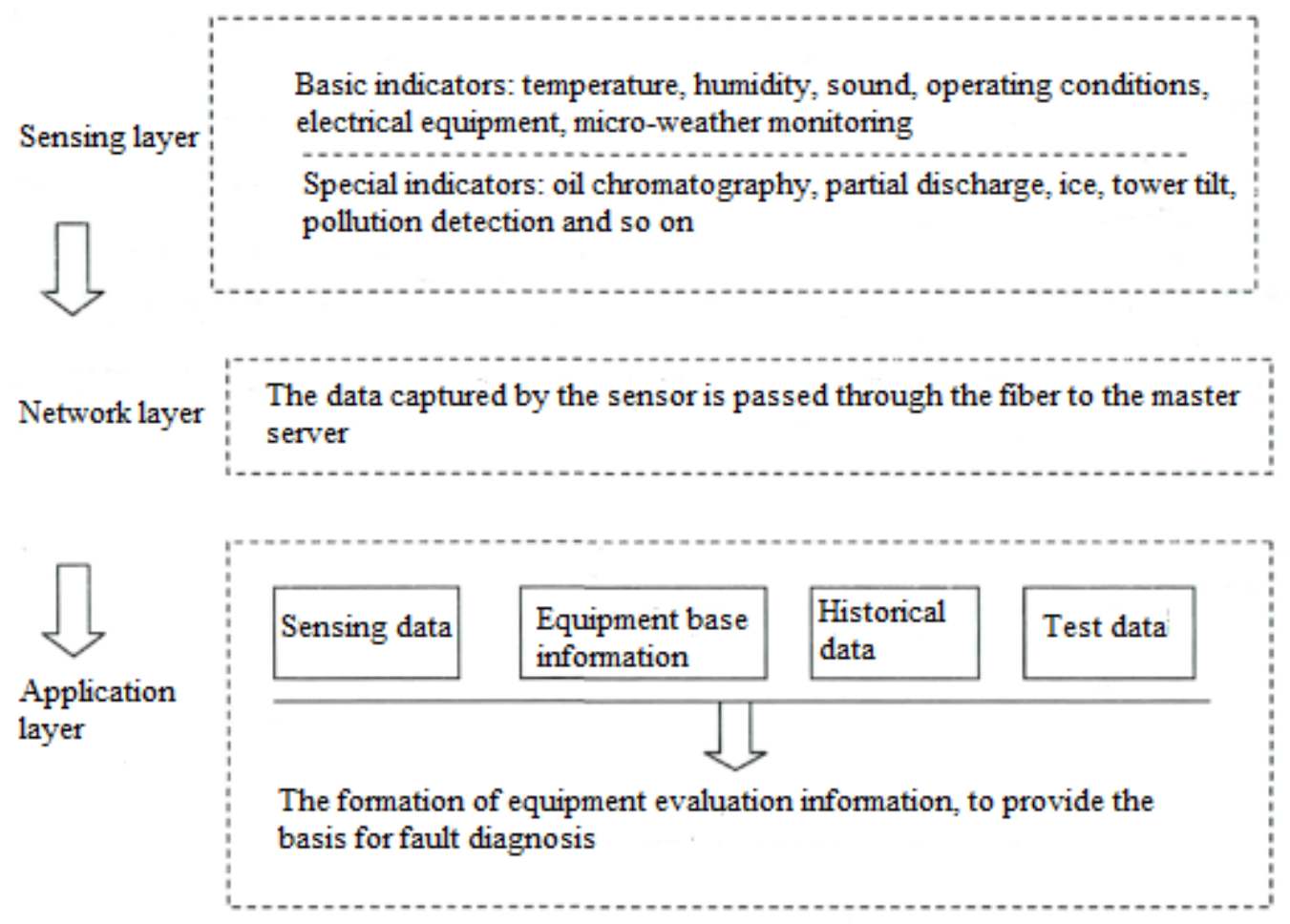

Figure 2 Based on the Internet of things technology maintenance diagram 
to adopt expert advice.

The simplified work diagram of the general maintenance plan is shown in Figure 1 below:

\subsection{Evaluation of the Application of Internet of Things Technology in State Maintenance}

After the in-depth study of the use of the Internet of Things technology in the state of power equipment, the technology can be found as a means of state maintenance, providing accurate and objective data support for subsequent information processing (see Figure 2 below). It can be seen that the state maintenance in addition to professional work, the need for certain management tools to data processing and decision-making as the cornerstone.

2. Application of Internet of Things Technology in Full-Cycle Management of Electric Power Equipment

For the full range of power equipment management aspects, the specific application of Internet of Things technology performance for the following:

Increase the initial cost of power system construction. Internet of Things technology is an invisible commodity, so its application will inevitably lead to the initial cost increase. However, with the advent of the digital information age, the cost has been included in the cost of some basic power equipment, so its impact is very small.

Delay the disposal of electrical equipment to a large extent. This article introduces the advantages of the Internet of Things technology in the state maintenance of electric power equipment that is, delaying the disposal of equipment, improving the working condition of the equipment and reducing the possibility of power failure.

Reduce human and financial resources. With the development of the information age, the role of labor in the power system will gradually become smaller, but the current individual labor costs are gradually increasing, and the higher risk of the power industry, resulting in annual failure due to human costs more. The application of Internet of Things technology to a large extent reduce labor costs, security maintenance, while improving the quality of power for the power companies to create more social and economic profits.

3. To the Internet of things based on state maintenance and general state maintenance comparison

This article on the Internet of things based on the state of maintenance to explore, and the need for general state maintenance comparison. According to the relevant literature, the following breakdown of the difference between the difference:

\subsection{All aspects of power system maintenance more in-} telligent

In terms of power transmission, the main object is the line part, such as reducing the transmission loss, improve the transmission efficiency, to ensure that the power and so on. Based on the Internet of Things technology can reduce the consumption of human and financial resources, such as China during the National Day parade, only Beijing has arranged thousands of power maintenance staff, and effective application in the Internet of things, can completely avoid this waste of resources.

In terms of the substation, with the domestic substation on duty in the form of maturity, the current monitoring method is difficult to adapt to high voltage, a wide range of substation needs, this time should be based on the Internet of Things to build monitoring network, the substation monitoring unified.

In terms of power distribution, according to the previous maintenance reports, lines and power equipment theft incidents occur more, simple manual monitoring cannot be real-time feedback. In addition, the phenomenon of stealing electricity is also worthy of attention. And these bad problems can be effected through the Internet of things to prevent and deal with state maintenance.

\subsection{The application of new intelligent sensing equip- ment}

In the intelligent power system, with a variety of new intelligent sensing facilities to ensure the accuracy of state maintenance information, because of any kind of analysis and processing technology, from the objective and correct information and data, are difficult to implement. General maintenance mode for the location of the movement, flow, speed and other state numerical measurements are often inaccurate, and based on the Internet of things technology is effectively improve the state of the problem.

Advanced sensing equipment, to achieve the information access and storage functions, and is an important prerequisite for data analysis, which is the implementation of the Internet based on the status of technology maintenance. For example, relay maintenance, the general state maintenance only by means of human inspection and equipment error report to achieve, the lack of sensor equipment, information, resulting in 
maintenance reports have time difference, increase the possibility of power accidents.

\subsection{Information transmission and storage of safe and convenient}

Traditional information transmission, storage is the use of cable to the electrical signal as the carrier, because the cable is very susceptible to electromagnetic interference, the safety is not high, long distance transmission by the humidity, temperature and other effects, the error rate is high, often caused by electrical signals Interrupted. And the Internet of Things technology to achieve the information transmission, storage, safe and convenient, because it usually with the cable transmission signal, the cable affected by the electromagnetic little or no information fidelity is better, more delivery, on the internal information transmission can also be wireless transmission which improve the real-time information. The information on the incoming master is a digital format that can be stored directly without the need for formatting and laying the foundation for information processing.

\subsection{Maintenance system of efficient and intelligent}

Based on the Internet of things maintenance system, including wireless communications, Internet and other forms of communication, to promote the concept of mobile communications, completely subvert the previous maintenance system. It summarizes all the substation equipment, for different types of equipment combined with the principle of maintenance work to develop maintenance programs and maintenance standards.
Now on the classification of substation equipment to take bar code technology, the work-flow is shown in Figure 3 below.

Compared with the previous maintenance means, the maintenance means to greatly enhance the efficiency, reduce the professional maintenance technology, the indirect role of accident liability, especially the new recruits, in its auxiliary maintenance, and slowly understand the power equipment maintenance work.

\subsection{Expert services}

Although the general state maintenance system also set up expert services, but because of the limitations of communication technology, did not achieve its function. For expert service characteristics, the objective accuracy of information directly affects the expert maintenance decision. However, the messyness of information hinders the setting of expert service function, and the weight method can solve the problem effectively, that is, measure the information with proportion, and improve the efficiency and quality of decision-making. For example, on the state of the substation equipment maintenance, the general transformer equipment failure at the same time, the proportion of gas also changes, the measurement of the experts accounted for greater weight, acceleration, displacement and other measurements despite the analysis of value, but In most cases because of measurement errors caused by, so the weight of less. In addition, the impact of different information on the transformer equipment failure, the current also changes, if the short time the current is relatively stable, but the gas

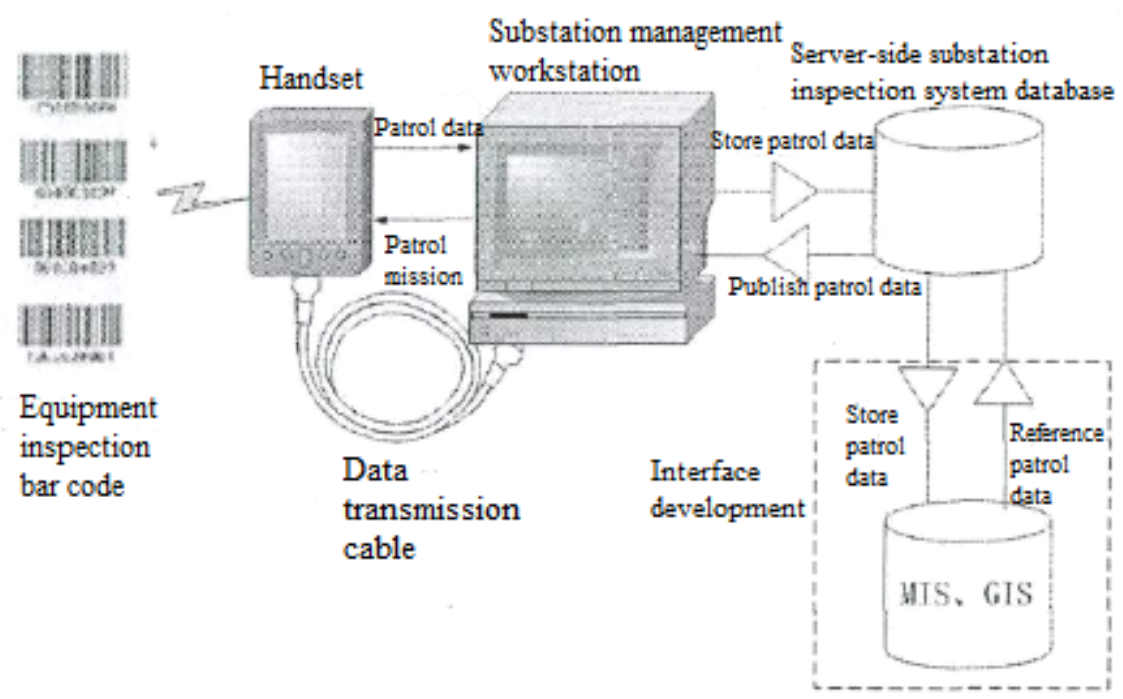

Figure 3 Maintenance system workflow 
changes significantly, it may be a serious failure of the transformer equipment.

\subsection{Reduce human resource consumption}

With the maturity of Internet of Things technology, a large extent to improve the efficiency of power equipment maintenance. Prior to the maintenance of electrical equipment is generally human detection, maintenance, especially in underdeveloped areas, maintenance work often consume more manpower and time. And because the special maintenance of electrical equipment, maintenance workers sometimes difficult to find a confirmation failure. So the Internet of Things technology to effectively improve the maintenance of electrical equipment in this area. Many sensors, Internet equipment applications, can be timely feedback basic data information, reducing human consumption, for the company to create more revenue. At the same time the technology also improved the failure of manpower maintenance problems, to ensure the quality of maintenance.

\subsection{Maintenance process is safe and reliable}

Most of the general state maintenance methods require staff to go to the scene to monitor the maintenance, and for some prone to dangerous accident maintenance, such as pressure equipment and oil spills, it is easy for maintenance personnel constitute a personal threat. In addition, because of the arbitrariness of manpower maintenance, once the work slack, then the power system problems will be difficult to real-time feedback. However, based on the state of the Internet technology maintenance, with the sensing device rather than human power will be able to detect failures, and real-time feedback to the maintenance staff, which is to prevent the deterioration of the fault in time. Thus, the application of Internet of Things technology in addition to ensuring the safety of staff, but also to protect the real-time power system failure on detection for effective prevention.

\section{Conclusion}

Power equipment status maintenance with the Internet of things to collect accurate and objective data information, but the data processing and maintenance of the program to determine no direct impact. Compared with the general state maintenance, based on the state of the Internet technology maintenance work with efficient and intelligent maintenance, maintenance process is safe and reliable, reduced human resources consumption, maintenance efficiency and other advantages, so on the Internet of things based on the status of power equipment maintenance further research has important practical significance and application value.

\section{References}

[1] Wei Baochun. Internet of Things Technical Characteristics and Application [J]. Automation and Instrumentation 2013 (04)

[2] Qu Tao-tao, Yan Fang .RFID and WSN Technology Integration Theory Research [J]. Internet of Things Technology.2013 (03)

[3] Qiao Zhenyi, Ren Jianwen, Wang Li, Ma Yezhi. Design and Implementation of Auxiliary Decision-Making System for Intelligent Equipment Maintenance [J]. Shaanxi Electric Power .2012 (11)

[4] Wang Jiangping, Chen Wei, Xiao Dong, Peng Bin, Xie Xiao. Design of Data Table Structure for Condition Maintenance Test Project of Power Transmission and Transformation Equipment [J]. Automation and Instrumentation 2013 (02)

[5] Wang Ping, Li Xiangping, Yan Dong, Zhu Baihan, Wang Ruitao. Design and Implementation of $2.4 \mathrm{GHz}$ Internet of Things Development Platform [J]. Automation \& Instrumentation 2012 (03)

[6] Chang Teng. Internet of Things technology development prospects [J]. Computer CD-ROM software and applications .2012 (13)

[7] Sheng Kuixiang. Talk about the Development and Application of Internet of Things Technology [J]. Modern business .2010 (14)

[8] Zhao Wei.Application and Exploration of Internet of Things Technology in Quantitative Management Platform of Scientific Research [J]. Automation and Instrumentation 2014 (06)

[9] Xie Jianrong, Chen Qingqi, Kuang Hongying, Duan Shousheng, Wang Lei, Huang Minjiang. Design of Infrared Temperature Online Monitoring Device for Power Equipment [J]. Automation \& Instrumentation.2012 (05)

[10] Jun Hou, Baowen Xu, Lei Xu, Di Wang, Junling Xu. A Testing Method for Web Services Composition Based on Data-flow [J].Wuhan University Journal of Natural Sciences.2008 (4)

[11] Andrew K.S. Jardine, Daming Lin, Dragan Banjevic. A Review on Machinery Diagnostics and Prognostics Implementing Condition-Based Maintenance [J]. Mechanical Systems and Signal Processing.2005 (7)

[12] Basim Al-Najjar, Imad Alsyouf. Selecting The Most Efficient Maintenance Approach Using Fuzzy Multiple Criteria Decision Making [J].International Journal of Production Economics.2002 (1) 